\title{
Utilising the Black Churches in the UK for health promotion: a nutrition intervention for first generation Ghanaian migrants in London
}

\author{
J. Adinkrah and D. Bhakta \\ Faculty of Life Sciences and Computing, London Metropolitan University, Holloway Road, London, N7 8DB, UK
}

Black and African Churches have been used successfully in the United States to deliver health promotion and interventions ${ }^{(1,2)}$. This study, to our knowledge, is the first in the UK to use the Black Churches setting as a vehicle for nutrition education and intervention. With the notion of churches been very important in the African community, it is hoped that this venue will aid in promoting and hopefully maintaining behavioural change in this population ${ }^{(3,4)}$.

A nutrition intervention programme was developed with input from focus groups of church congregants and the Obesity Clinic at the London Metropolitan University ${ }^{(4)}$. Participants were from two churches in Tower Hamlets and Newham. The church in Tower Hamlets served as the control and the other as the intervention. The control group were provided with a booklet on healthy eating, whereas the intervention group were asked to attend six sessions of nutrition education every week at the Church. The sessions included information on portion size control, eating out etc etc. Baseline measurements of anthropometry, socio-demography and dietary intake were taken and repeated at 6 weeks and 3 months. Dietary intake was assessed using a validated FFQ ${ }^{(5)}$.

Table 1. Body composition changes at 3 months

\begin{tabular}{|c|c|c|c|c|c|c|c|c|c|c|}
\hline \multirow[b]{2}{*}{ Outcome measures } & \multicolumn{5}{|c|}{ Intervention $(n=23)$} & \multicolumn{5}{|c|}{ Control $(n=19)$} \\
\hline & Base line & SD & 3 months & SD & $P$ value & Base line & SD & 3 months & SD & $P$ value \\
\hline Weight (kg) & 72.6 & 1.3 & 70.3 & 8.7 & 0.001 & 68.1 & 3.8 & 67.2 & 2.0 & 0.023 \\
\hline BMI $\left(\mathrm{kg} / \mathrm{m}^{2}\right)$ & 27.5 & 0.5 & 26.1 & 3.0 & 0.001 & 25.5 & 1.2 & 24.4 & 1.0 & 0.056 \\
\hline Waist circum. $(\mathrm{cm})$ & 91.6 & 1.2 & 88.6 & 1.6 & 0.003 & 90.1 & 5.6 & 88.5 & 4.2 & 0.017 \\
\hline Body fat $(\%)$ & 29.2 & 2.3 & 27.2 & 2.1 & 0.001 & 27.1 & 3.9 & 26.4 & 4.4 & 0.060 \\
\hline
\end{tabular}

Table 2. Dietary intake changes at 3 months

\begin{tabular}{|c|c|c|c|c|c|c|c|c|c|c|}
\hline \multirow[b]{2}{*}{ Outcome measures } & \multicolumn{5}{|c|}{ Intervention $(n=23)$} & \multicolumn{5}{|c|}{ Control $(n=19)$} \\
\hline & Base line & SD & 3 Months & SD & $P$ value & Base line & SD & 3 Months & SD & $P$ value \\
\hline Energy (g) & 2022 & 282.4 & 1976 & 119.3 & 0.147 & 2072 & 384.5 & 2058 & 365 & 0.237 \\
\hline Protein (g) & 67 & 24.4 & 70 & 19.3 & 0.079 & 70 & 30.0 & 67 & 15.9 & 0.596 \\
\hline Fat $(\mathrm{g})$ & 76 & 37.1 & 69 & 39.0 & 0.017 & 84 & 8.0 & 73 & 24.5 & 0.001 \\
\hline Carbohydrate (g) & 268 & 23.2 & 269 & 56.9 & 0.580 & 280 & 96.7 & 274 & 67.2 & 0.377 \\
\hline Fibre $(\mathrm{g})$ & 17.1 & 4.5 & 18.9 & 3.7 & 0.074 & 16.9 & 5.0 & 14.2 & 3.9 & 0.072 \\
\hline
\end{tabular}

We found that both the intervention and control group showed favourable changes of decrease in body weight, BMI, waist circumference and percentage body fat. The changes were significant for body fat and BMI for the intervention group at 3 months (Table 1). Energy, fat and salt intake also decreased in both groups (Table 2).

These preliminary findings show that a culturally sensitive church-based intervention programme can be an innovative way to educate and promote a healthier lifestyle.

1. Clay KS, Newlin K \& Leeks KD (2005) Can, Cult and Lit, pp 111-115.

2. Campbell MK, Motsinger BM, Ingram A, Jewell D et al. (2000) H Educ Behav 27(2), 241-53.

3. Reynolds KD, Klepp K and Yaroch AL (2004) Nutrition Society pp, 106-119.

4. Resnicow K, Campbell MK, Carr C et al. (2004) Am J Prev Med 27, 97-105.

5. Adinkrah J \& Bhakta D (2010) Proc Nutr Soc 69(OCE1), E37. 